\title{
Management of venous thromboembolism in patients with cancer: role of dalteparin
}

\author{
Lori-Ann Linkins
}

Department of Hematology and Thrombosis, McMaster University, Hamilton, Ontario, Canada
Correspondence: Lori-Ann Linkins Henderson Hospital, 7I I Concession Street, Hamilton, ON L8V IC3, Canada Tel + I 90538944 I I

Fax + I 9055747625

Email llinkins@thrombosis.hhscr.org

\begin{abstract}
Cancer is a major risk factor for the development of venous thromboembolism (VTE). Conventional anticoagulant therapy with a vitamin $\mathrm{K}$ antagonist is more problematic in cancer patients due to an increased risk of recurrent VTE, and an increased risk of anticoagulant-related bleeding. In recent years, there has been a shift toward treating cancer patients with VTE with extended duration dalteparin. Dalteparin, a low-molecular-weight heparin, has been shown to be more effective, and as safe as conventional anticoagulant therapy, in cancer patients with VTE. This paper will (a) review the relationship between cancer and VTE, and (b) provide an overview of the role of dalteparin in the management of VTE in patients with cancer.
\end{abstract}

Keywords: dalteparin, cancer, venous thromboembolism

\section{Introduction}

Cancer is a major risk factor for the development of venous thromboembolism (VTE). Patients with cancer have a 4- to 7-fold higher incidence of VTE than patients without cancer (Heit et al 2000b; Blom et al 2005) Cancer is also a major risk factor for bleeding. Patients with cancer and VTE have a 2-fold higher risk of major bleeding while receiving anticoagulant therapy than patients with VTE who do not have cancer (Prandoni et al 2002). Adding further complexity to the issue, patients with cancer and VTE who are treated with conventional anticoagulant therapy are more likely to develop recurrent VTE than their non-cancer cohorts. To better balance the need for efficacy with safety, anticoagulant therapy for patients with VTE and cancer has recently shifted away from conventional treatment to long-term therapy with the lowmolecular weight heparin, dalteparin (Fragmin ${ }^{\circledR}$; Pfizer). This paper will (a) review the relationship between cancer and VTE, and (b) provide an overview of the role of dalteparin in the management of VTE in patients with cancer.

\section{Cancer and venous thromboembolism \\ Risk}

The incidence of VTE in patients with cancer has been difficult to determine because of the heterogeneity of the patient population. The most recent estimates come from two large population-based studies which linked cancer registries to other healthrelated databases (Blom et al 2006; Chew et al 2006). Blom and colleagues linked a Netherlands cancer registry to regional anticoagulation clinics $(n=66,329$ patients $)$ and reported a cumulative incidence of VTE of 24.6 per 1000 patients in the first year following cancer diagnosis (Blom et al 2006). Chew and colleagues linked a California cancer registry to a state-wide patient discharge database $(n=235,149$ patients $)$ and reported a cumulative incidence of VTE of 16 per 1000 patients over the first 2 years following cancer diagnosis (Chew et al 2006). Although these registries consist of a large number of patients, it is likely that they underestimate the incidence of VTE in 
patients with cancer because not all cases were reported. The reported incidence of VTE in smaller cancer cohort studies ranges from $7.8 \%$ (ie, deep vein thrombosis [DVT] and pulmonary embolism [PE] in patients with solid tumors) to $13.6 \%$ (ie, DVT only in patients with non-small cell lung cancer) (Sallah et al 2002; Tagalakis et al 2007).

A recent retrospective review of 435 consecutive staging CT scans performed on patients with a variety of tumor types reported a prevalence of clinically silent VTE of $6.3 \%$ (Cronin et al 2007). These data suggests that the true incidence of VTE in cancer patients is likely much higher than indicated by any of the previous studies.

The major determinants of the risk of VTE in cancer patients include tumor stage, tumor type, chemotherapy, hormonal therapy, surgery, and presence of central venous catheters. Patients with metastatic cancer have at least a 2- to 4-fold increased risk of VTE in the first 6 months after cancer diagnosis compared to patients with limited stage cancer (Blom et al 2005, 2006). Chew and colleagues reported that the patients with metastatic cancer who have the highest incidence of VTE are as follows: pancreatic cancer (20 per 100 patient-years), stomach (10.7 per 100 patient-years), bladder (7.9 per 100 patient-years), uterine (6.4 per 100 patientyears), renal (6.0 per 100 patient-years), and lung (5.0 per 100 patient-years) (Chew et al 2006). The true incidence of VTE according to tumor type is controversial because of the heterogeneity of the population. In one report, the tumor types with the highest risk of VTE were bone, ovary, brain, and pancreas (Blom et al 2006). In the same report, it was noted that more cases of VTE were observed in patients with tumors of the breast, prostate, lung and colon, presumably because these types of cancer have a higher prevalence and a longer survival time (Blom et al 2006).

The risk of VTE in patients receiving chemotherapy is a moving target because of the variety of agents currently available as well as the ongoing introduction of new agents. In one retrospective review of 206 unselected cancer patients who received chemotherapy, the annual incidence of VTE was reported to be $11 \%$ (Otten et al 2004). Similar estimates have been reported by other investigators for patients with breast cancer (von Tempelhoff et al 1996) and ovarian cancer (von Tempelhoff et al 1998). The risk of VTE has been noted to be significantly higher in patients who receive a combination of agents. For example, the incidence of VTE when patients with multiple myeloma are given thalidomide or dexamethasone alone is increased by 2.6-fold and 2.8-fold, respectively. When these agents are given in combination, the risk of VTE rises to 8-fold (Haddad and Greeno 2006;
El Accaoui et al 2007). Similarly, the combination of tamoxifen with chemotherapy in the treatment of women with breast cancer increases the risk 3 to 5-fold higher than with tamoxifen alone (Deitcher and Gomes 2004).

The risk of VTE has been brought to the forefront as a significant safety concern during evaluation of some of the newer antineoplastic agents. Lenalidomide, an analog of thalidomide, has fewer side effects than its predecessor, but shares its high risk of VTE (ranging from $4 \%$ to $75 \%$ in uncontrolled studies) (Hirsh 2007). This observation has prompted investigators of one ongoing trial to mandate that all study subjects in the lenalidomide-high dose dexamethasone arm receive prophylaxis with aspirin, low-molecular-weight heparin or warfarin (Rajkumar and Blood 2006; Zonder et al 2006). (There are no randomized controlled trials to support the effectiveness of aspirin in this setting [Hirsh 2007].) With another agent, bevacizumab (an angiogenesis inhibitor), initial reports suggested that the risk of VTE in patients was a major concern (Kabbinavar et al 2003; Shah et al 2005), but more recent studies have shown that it is likely the combination of agents, and not bevacizumab alone, that leads to the high rate of thrombosis (Herbst and Sandler 2004; Hurwitz et al 2004; Rugo 2004; Haddad and Greeno 2006)

Supportive agents used to treat cancer patients, such as erythropoietin, granulocyte colony-stimulating factor, and high-dose corticosteroids have also been implicated as risk factors for VTE in cancer patients (Haddad and Greeno 2006).

Surgery and the use of indwelling central venous catheters are risk factors for VTE in the general population. The addition of cancer under these conditions leads to a cumulative increase in risk. The risk of post-operative VTE in cancer patients who undergo surgery is at least double the risk for non-cancer patients who undergo the same procedure (Geerts et al 2004). More importantly, cancer patients who undergo surgery have a 3-fold higher risk of fatal PE, as confirmed on autopsy, compared with non-cancer patients (Kakkar et al 2005).

Central venous catheters are essential for the management of many cancer patients, but they also increase the risk of upper limb venous thrombosis and pulmonary embolism. The incidence of symptomatic catheter-rated thrombosis had previously been reported to be as high as $28 \%$, but more recent studies place the risk closer to $4 \%$ (Lee et al 2006). Risk factors for the development of catheter-related thrombosis in one prospective study included multiple insertion attempts, previous central venous catherization, and ovarian cancer (Lee et al 2006). 
The diagnosis of VTE in a cancer patient is associated with a poor outcome. These patients have a 2- to 4-fold increased risk of recurrent VTE while receiving anticoagulant therapy as compared with non-cancer patients with VTE (Heit et al 2000a; Prandoni et al 2002). They also have 2-fold higher risk of major bleeding (Prandoni et al 2002). Unlike non-cancer patients with VTE, most thrombotic and bleeding complications in patients with cancer and VTE occur while anticoagulant therapy is within the established therapeutic range (Hutten et al 2000; Prandoni et al 2002). Lastly, cancer patients with VTE have a higher mortality rate than cancer patients who do not have VTE (hazard ratio 1.6-4.2, p < 0.01) (Chew et al 2006). Mortality appears to be particularly high in patients who have cancer diagnosed at the same time as their primary VTE (ie, 1-year survival rate of $12 \%$ compared with $36 \%$ survival rate in patients without VTE who were matched for type of cancer, age, sex, and year of diagnosis) (Sorensen et al 2000).

\section{Pathogenesis}

Cancer promotes the development of VTE by inducing a hypercoaguable state. The mechanisms by which this hypercoaguable state is produced are complex and multifactorial. It has been shown that tumor cells promote activation of blood coagulation by: (i) producing procoagulant factors (Gale and Gordon 2001), (ii) releasing cytokines (Grignani and Maiolo 2000), and (iii) by direct cell-to-cell interaction with patient endothelial cells, leucocytes and platelets (Prandoni et al 2005; Buller et al 2007). One of the key procoagulant factors produced is tissue factor, the primary activator of normal blood coagulation. While normal endothelial cells only express tissue factor when stimulated, tumor cells constitutively express tissue factor. An increased level of tissue factor promotes angiogenesis, and permeability of the vascular endothelium to tumor cells. Another procoagulant factor produced by tumor cells is cancer procoagulant, a cysteine proteinase that activates Factor $\mathrm{X}$ independently of Factor VIIa. Cytokines produced and released by tumor cells include TNF $\alpha$, IL-1 $\beta$, and vascular endothelial growth factor (VEGF). TNF $\alpha$ and IL-1 $\beta$ promote thrombosis at the vascular wall by inducing endothelial expression of tissue factor and downregulating expression of thrombomodulin, the endothelial thrombin receptor that plays a key role in the activation of the anticoagulant protein $\mathrm{C}$ pathway. Increased levels of VEGF contribute to angiogenesis and inhibit apoptosis. Lastly, tumor cells express cell-adhesion molecules on their surface, which allows them to directly interact with endothelial cells, platelets, and leucocytes. The end result of the mechanisms above, and others not outlined in this review, is the promotion of fibrin formation. Fibrin formation, the final step in the blood coagulation pathway, has been shown to support angiogenesis which, in turn, promotes tumor growth and metastasis (Bromberg et al 1995; Rickles et al 2003; Rak et al 2006).

\section{Prevention}

There is strong evidence to support the need for primary thromboprophylaxis in patients who undergo surgery or require prolonged hospitalization (Geerts et al 2004). Both unfractionated heparin and low-molecular-weight heparin, administered subcutaneously in low doses, have been shown to be effective and safe for thromboprophylaxis in these settings in the general population (Geerts et al 2004). Although the majority of data for thromboprophylaxis for cancer patients comes from subgroup analyses of general surgery studies (Mismetti et al 2001; Bergqvist 2007), there are a few studies which restricted enrolment to cancer patients. In the randomized, double-blind ENOXACAN study, enoxaparin (Lovenox $^{\circledR}$; Sanofi-Aventis) 40 mg once-daily (started 2 hours pre-operatively) was compared with unfractionated heparin 5000 units 3 times daily in 1115 high-risk patients over 40 years of age who underwent elective curative abdominal or pelvic surgery for cancer (ENOXACAN Study Group 1997). The incidence of VTE (on bilateral venography or pulmonary scintigraphy) and bleeding rates were equivalent in the two groups. In a smaller study, dalteparin 5000 units daily (starting with a 2500 unit dose 2 hours pre-operatively and another 12 hours postoperatively) was compared with unfractionated heparin 5000 units 3 times daily (starting with a dose 2 hours pre-operatively) in 40 patients undergoing pelvic or abdominal cancer surgery (Fricker et al 1988). Both regimens were found to be equally effective and safe.

The efficacy and safety of extended duration thromboprophylaxis in cancer patients undergoing surgery has been evaluated in 4 studies to date ( 2 published as papers and 2 published in abstract form) (Rasmussen et al 2005). ENOXACAN II was a randomized double-blind study that compared 1 week of thromboprophylaxis with enoxaparin $40 \mathrm{mg}$ daily with a 4-week course of enoxaparin in patients undergoing elective surgery for abdominal or pelvic cancer surgery (Bergqvist et al 2002). The results showed a 60\% reduction in the relative risk of venographically detected VTE with the 4-week course compared with the 1 week course ( $4.8 \%$ vs $12 \%, p=0.02)$. The incidence of major bleeding was not significantly different between the two groups. Similar results were reported in an open label study that compared 
dalteparin 5000 units once daily for 7 days with the same regimen for 28 days in 343 patients ( 200 patients with cancer) who underwent major abdominal surgery (Rasmussen et al 2006). The primary outcome, venographically-detected VTE, occurred in $16.3 \%$ in the short-term prophylaxis arm and $7.3 \%$ in the extended duration prophylaxis arm (relative risk reduction $55 \%, \mathrm{p}=0.012$ ). Current guidelines recommend extended duration thromboprophylaxis of 28-35 days in cancer patients who undergo high-risk surgery (eg, orthopedic surgery) (Geerts et al 2004).

Both warfarin and low-molecular-weight heparin have been evaluated for primary thromboprophylaxis in patients with indwelling central venous catheters (Couban et al 2005; Verso et al 2005; Karthaus et al 2006). Neither agent was found to be effective at preventing symptomatic catheterrelated VTE and neither agent is currently recommended for this indication (Geerts et al 2004).

\section{Management}

Conventional treatment of VTE consists of 5-7 days of therapeutic dose heparin or low-molecular-weight heparin followed by a vitamin $\mathrm{K}$ antagonist for a minimum of 3 months titrated to an international normalization ratio (INR) of 2.0-3.0. Unfortunately, conventional anticoagulant therapy in VTE patients who also have cancer tends to be more problematic than in non-cancer patients with VTE. For example, the long half-life of vitamin $\mathrm{K}$ antagonists creates management difficulties in cancer patients who require frequent invasive procedures (eg, therapeutic paracentesis) and develop chemotherapy-induced thrombocytopenia. Cancer patients also tend to have poor appetites and take multiple medications, both of which lead to erratic INRs and difficulties with warfarin dosing. Finally, cancer patients who require frequent needle sticks for the administration of chemotherapy, also tend to have poor venous access, which can make INR monitoring in the community a challenge (Lee and Levine 2003).

Recently, studies have shown that extended duration low-molecular-weight heparin is an effective and safe alternative to conventional anticoagulant therapy in cancer patients with VTE. The first published study was CANTHANOX, an open-label comparison of 3 months of warfarin with enoxaparin in 146 cancer patients with proximal DVT, PE, or both (Meyer et al 2002). This trial was terminated early due to poor recruitment. The investigators reported no significant difference in the incidence of the primary outcome, a combined endpoint of major bleeding or recurrent VTE within 3 months.
The second study was LITE, a randomized multicentre trial that compared warfarin with tinzaparin (Innohep ${ }^{\circledR}$; Leo Pharma) in 737 patients with proximal DVT (200 patients had cancer). (Hull et al 2006) After 12 weeks of treatment, recurrent VTE occurred in $10 \%$ of the cancer patients who received warfarin, and $6 \%$ of the cancer patients who received tinzaparin. One year after randomization, 16\% of cancer patients who received warfarin had recurrent VTE, in comparison with $7 \%$ of cancer patients who received tinzaparin $(p=0.044)$. There was no significant difference between the groups with respect to bleeding (7\% major bleeds in both groups) or mortality (19\% mortality in warfarin group versus $20 \%$ mortality in tinzaparin group) at 3 months.

The third study was ONCENOX, an open-label comparison of warfarin with two different doses of enoxaparin in 101 cancer patients with acute VTE (Deitcher et al 2006). This trial was terminated early due to poor recruitment. After 6 months of treatment, recurrent VTE occurred in $10.3 \%$ of patients who received warfarin, $6.9 \%$ of patients who received low-dose enoxaparin, and $6.3 \%$ of patients who received higher-dose enoxaparin. Major bleeding occurred in $2.9 \%$ of patients who received warfarin, $6.5 \%$ of patients who received low-dose enoxaparin, and $11.1 \%$ of patients who received high-dose enoxaparin ( $\mathrm{p}=$ not significant). The mortality rate was $32.4 \%$ of patients who received warfarin, $22.6 \%$ of patients who received low-dose enoxaparin, and $41.7 \%$ of patients who received high-dose enoxaparin ( $\mathrm{p}=$ not significant $)$.

Early termination of both the CANTHANOX and ONCENOX trials means that neither study was powered to show a significant difference in efficacy or safety between warfarin and low-molecular-weight heparin in cancer patients. Poor recruitment into these studies, possibly due to the requirement for injections, suggested that adequately powered trials to study this issue might not be possible. However, two further studies evaluating extended duration low-molecular-weight heparin (dalteparin) for treatment of cancer-related VTE were successfully completed and will be discussed in detail later in this review (Lee et al 2003; Monreal et al 2004).

The duration of anticoagulant therapy in cancer patients with VTE is also problematic. It is generally accepted that patients with metastatic cancer should remain on anticoagulants indefinitely, unless they develop bleeding complications or request discontinuation for quality-of-life reasons. However, the appropriate duration of anticoagulant therapy in patients with limited stage cancer (I or II) who develop VTE is unknown. If the malignancy is in remission, and other 
risk factors have resolved (eg, immobility, chemotherapy, surgery), it is likely safe to discontinue anticoagulants after a minimum of 3 months of treatment. However, this strategy has never been evaluated in a clinical trial, and there is always the risk that recurrent VTE might be the first clinical sign of return of the malignancy.

\section{Role of dalteparin in the management of venous thromboembolism in cancer patients \\ Pharmacology and pharmacokinetics}

Dalteparin sodium (Fragmin ${ }^{\circledR}$; Pfizer) is a low-molecular-weight heparin that consists of strongly acidic sulphated polysaccharide chains with a mean molecular weight of $5000 \mathrm{Da}$ (range 2000-9000 Da). It is derived from pork intestinal mucosal heparin (molecular weight 3000-30,000 Da) by partial nitrous acid depolymerization (Fareed et al 2004). Similar to unfractionated heparin, dalteparin contains the pentasaccharide sequence that is responsible for its ability to bind to antithrombin. In contrast to unfractionated heparin, the majority of dalteparin chains do not contain the 18 saccharide sequence that is required to simultaneously bind to antithrombin and thrombin. Consequently, dalteparin is able to induce the conformational change in antithrombin that enhances its ability to inhibit activated coagulation factors Xa (also IXa, XIa, XIIa), but it is not able to enhance antithrombin-dependent inhibition of thrombin (ie, reduced anti-IIa/anti-Xa ratio). The anticoagulant effect of dalteparin is, by convention, expressed in terms of its anti-Xa activity (international standard units [IU] per kg). The specific activity of dalteparin on factor $\mathrm{Xa}$ is $130 \mathrm{IU} / \mathrm{mg}$ and on thrombin is $58 \mathrm{IU} / \mathrm{mg}$ therefore the anti-IIa/anti-Xa ratio for dalteparin is 2.2 (Anonymous 2006).

The goal for the development of dalteparin and the other low-molecular-weight heparins was to overcome the pharmacokinetic limitations of unfractionated heparin (referred to as heparin for the remainder of this review) (Hirsh and Raschke 2004). Firstly, heparin binds to plasma proteins, which produces an unpredictable anticoagulant response, and makes laboratory monitoring necessary when it is given intravenously. Secondly, it has poor subcutaneous bioavailability $(20 \%-30 \%)$ and a short half-life which means it must be administered twice daily when given by subcutaneous injection. Finally, heparin binds to platelet factor IV (PF4) which reduces its anticoagulant effect, and can lead to the development of heparin-induced thrombocytopenia (HIT).
In contrast, dalteparin does not bind to plasma proteins, has excellent bioavailability (87\%) when given by subcutaneous injection (Bratt et al 1986), and produces a predictable anticoagulant response (Hirsh and Raschke 2004). HIT has been reported in patients who received dalteparin, but the incidence is estimated at $1 \%$ versus $12 \%$ with heparin (Levine et al 2006).

Dalteparin exhibits dose-independent first order pharmacokinetics (Dunn and Jarvis 2000). The rate-limiting step after subcutaneous injection is absorption (Bratt et al 1986). Peak plasma concentrations are reached 3-5 hours after subcutaneous injection (Handeland et al 1990). The volume of distribution in healthy volunteers was 7.7-9 L (Collignon et al 1995). The plasma elimination half-life is $3-4$ hours (Simoneau et al 1992; Collignon et al 1995) The principle route of elimination of dalteparin is renal. The product monograph for dalteparin currently recommends monitoring and dose adjustment for patients with renal insufficiency (especially for patients with a creatinine clearance of less than $30 \mathrm{~mL} / \mathrm{min}$ ) due to concern about prolongation of anti-Xa activity. However, a recent pilot study has shown that when given in prophylactic doses (5000 units daily) to intensive care unit patients with renal insufficiency, peak anti-Xa levels remained within the conventional prophylactic range (Rabbat et al 2005). Monitoring of anti-Xa levels in standard risk patients who are given body-weight adjusted doses of dalteparin is not necessary (Alhenc-Gelas et al 1994; Boneu and de Moerloose 2001; Hirsh and Raschke 2004). A substudy of cancer patients who received extended duration dalteparin at therapeutic doses to treat VTE did not show any evidence of bioaccumulation after one month of treatment (Kovacs et al 2005).

\section{Treatment of venous thromboembolism}

Subcutaneous dalteparin has been compared with heparin for initial treatment of VTE in multiple randomized trials (Holm et al 1986; Harenberg et al 1990; Lindmarker et al 1994; Meyer et al 1995; Fiessinger et al 1996; Luomanmaki et al 1996; Kearon et al 2006). The consensus is that dalteparin and the other low-molecular-weight heparins are safe and effective as conventional anticoagulant therapy for treatment of acute VTE (Leizorovicz et al 1994; Gould et al 1999; Quinlan et al 2004). Only one head-to-head trial comparing dalteparin with another low-molecular-weight heparin has been conducted in this patient population. Wells and colleagues randomized 254 patients with acute VTE to receive either dalteparin $200 \mathrm{IU} / \mathrm{kg}$ or tinzaparin $175 \mathrm{IU} / \mathrm{kg}$ for a minimum of 5 days followed by a vitamin antagonist for 3 months (Wells et al 2005). The primary outcome, a composite of recurrent VTE and bleeding, 
occurred in $4.4 \%$ of the patients who received dalteparin and $5.9 \%$ of the patients who received tinzaparin $(p=0.44)$.

\section{Treatment of venous thromboembolism in cancer patients}

Two clinical trials and one case series evaluating extended duration dalteparin for treatment of VTE in cancer patients have been published to date.

\section{CLOT}

The CLOT study was a 676 patient open-label trial that randomized cancer patients with acute DVT, PE, or both to conventional anticoagulant therapy or extended duration dalteparin (Lee et al 2003). Patients in the conventional treatment arm were given 5-7 days of dalteparin $200 \mathrm{IU} / \mathrm{kg}$ (maximum 18,000 IU) by once-daily subcutaneous injection followed by a vitamin K antagonist for 6 months (INR 2.0-3.0). Patients in the extended duration dalteparin arm were given dalteparin $200 \mathrm{IU} / \mathrm{kg}$ (maximum 18,000 IU) by once-daily subcutaneous injection for the first 4 weeks followed by dalteparin $150 \mathrm{IU} / \mathrm{kg}$ for the remaining 5 months.

The primary outcome measure, symptomatic recurrent VTE at 6 months, occurred in 27 patients (8\%) in the extended duration dalteparin arm, and 53 patients (15.8\%) in the conventional treatment arm (relative risk reduction $52 \%$; $\mathrm{p}=0.002$ ). There were 5 fatal PEs in the extended duration dalteparin arm, and 7 fatal PEs in the conventional treatment arm. The majority of recurrent VTE in the conventional treatment arm occurred when the INR was within or above the therapeutic range.

Major bleeding occurred in 19 patients $(6 \%)$ in the extended duration dalteparin arm and 12 patients $(4 \%)$ in the conventional treatment $\operatorname{arm}(\mathrm{p}=0.27)$. There was 1 fatal bleed in the extended duration dalteparin arm and no fatal bleeds in the conventional treatment arm. At the time of a major bleeding event, 2 patients in the extended duration dalteparin arm had thrombocytopenia, and 6 patients in the conventional treatment arm had an INR greater than 3.0. The overall mortality rate did not differ significantly between the two groups. The authors concluded that extended duration dalteparin was more effective, and as safe as conventional anticoagulant therapy for patients with cancer and acute VTE. A comparison of the properties of warfarin with extended duration dalteparin for long-term anticoagulation is given in Table 1 .

\section{Monreal cohort study}

The second trial was a prospective cohort study of 203 patients with metastatic cancer and symptomatic VTE (Monreal et al 2004). Patients received dalteparin $200 \mathrm{IU} / \mathrm{kg}$ subcutaneously once daily for 7 days followed by a fixed dose of dalteparin 10,000 IU once daily for at least 3 months. During the 3 month study period, 11 patients (5.4\%) developed major bleeding complications, 6 of which were fatal. Recurrent VTE occurred in 21 patients (10.3\%), 2 of which were fatal PEs. Three patients with recurrent VTE had their thrombotic event shortly after adjustment of their dalteparin dose in response to a bleeding event. The authors concluded that fixed dose dalteparin for 3 months does not increase bleeding in cancer patients with acute VTE and liver or brain metastases.

\section{Noble case series}

Noble and colleagues reported on a case series of 62 palliative care cancer patients who received dalteparin for treatment of VTE (Noble et al 2007). None of the patients in this series were receiving active antineoplastic treatment at the time of treatment of their VTE, and $95 \%$ had metastatic disease (the remaining $5 \%$ had incurable primary brain malignancy). ECOG status and life expectancy were not reported. Dalteparin was given to the patients according to either the CLOT study

Table I Comparison of warfarin with dalteparin for long-term anticoagulation

\begin{tabular}{|c|c|}
\hline Warfarin & Extended duration dalteparin \\
\hline 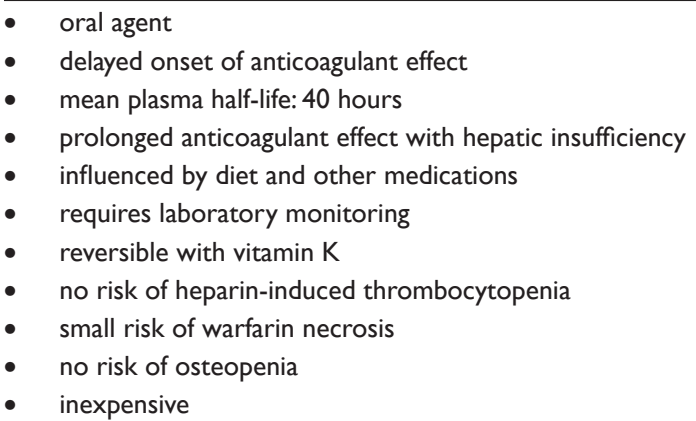 & 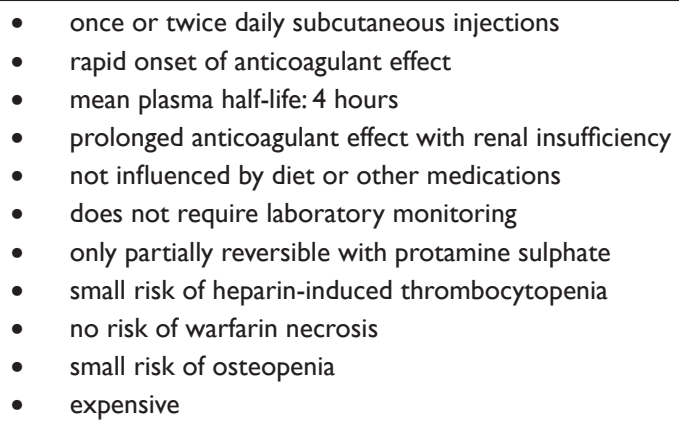 \\
\hline
\end{tabular}


(Lee et al 2003) or Monreal protocol (Monreal et al 2004). The median duration of dalteparin treatment was 97 days (range 23-243 days). The injections were administered by the patient $(n=46)$, a caregiver $(n=15)$ or a nurse $(n=1)$. Three patients had a clinical suspicion of recurrent VTE while off of dalteparin (only 1 event was confirmed). Five patients had a minor bleed, of which only 1 patient stopped dalteparin (3 patients with bleeds were switched from the CLOT protocol to the Monreal protocol). Fifty patients stopped dalteparin treatment due to commencement of end of life pathway, and 7 stopped dalteparin after completion of 6 months of treatment ( 3 of these patients were clinically suspected to have recurrent VTE while off of treatment). The authors concluded that within the limitations of a case series, low-molecular-weight heparin appears to be effective in the setting of palliative advanced cancer.

No head to head trials comparing different types of lowmolecular-weight heparins in the treatment of cancer-related VTE have been published to date.

\section{Tolerability and quality-of-life with dalteparin}

Despite the requirement for subcutaneous injections, there is evidence that dalteparin is generally well tolerated by cancer patients. In the CLOT study, only $6 \%$ patients withdrew from the extended duration dalteparin arm because of the injections (in comparison, $4 \%$ patients withdrew from the conventional treatment arm) (Hull and Hull 2003). Noble and colleagues evaluated acceptability of low-molecular-weight heparin to 40 palliative care cancer patients using semi-structured interviews (Noble and Finlay 2005). They found that patients preferred the freedom from blood tests that low-molecular-weight offered them over conventional anticoagulation with a vitamin $\mathrm{K}$ antagonist. Although many of the patients reported discomfort at the point of the injection, they described it as "short-lived" and did not label it as a significant deterrent to continuing the injections. Acceptability of low-molecular-weight injections by cancer patients who do not have VTE (ie, cancer patients in clinical trials evaluating the potential survival advantage of low-molecular-weight heparin) has not been reported.

\section{Economic implications of extended duration dalteparin treatment}

Despite recommendations based on the results of the CLOT trial (Buller et al 2004), extended duration dalteparin treatment of cancer-related VTE has not been widely adopted (pers comm). The most significant barrier to adoption of this treatment is cost. Two different groups have recently conducted studies evaluating the economic implications of extended duration low-molecular-weight heparin treatment in patients with cancer-associated VTE. The findings from these studies are outlined below (Aujesky et al 2005; Dranitsaris et al 2006).

Using a decision analytic model, Aujesky et al performed a cost-effectiveness analysis for a hypothetical cohort of 65 -year-old cancer patients receiving a 6 month course of low-molecular-weight heparin compared with warfarin (Aujesky et al 2005). This analysis, performed from the US societal perspective, compared anticoagulant strategies based on quality-adjusted life-years (QALYs) and lifetime costs. They determined that the low-molecular-weight heparin strategy achieved a higher incremental quality-adjusted life expectancy than warfarin (by 0.051 QALYs), but at a lifetime cost increment of US\$7609 (nearly double the cost of warfarin). The cost of low-molecular-weight heparin in this model was based on 2002 US prices and on $20 \%$ of patients requiring nursing visits to administer injections. The authors concluded that LMWH is more effective than warfarin, but at a significantly higher cost.

Dranitsaris et al performed a pharmacoeconomic analysis to determine whether extended duration dalteparin in patients with cancer-related VTE was an economically reasonable alternative to warfarin (Dranitsaris et al 2006). This analysis, performed from the perspective of the publicly funded Canadian healthcare system, used the data from the CLOT trial to derive the overall cost of both anticoagulant strategies. The cost difference between the two strategies was used to determine incremental cost per QALY gained with dalteparin. They reported that the mean cost per patient treated with dalteparin for 6 months was CAN \$4162 (2005) compared with warfarin at CAN\$2003 (2005). The dalteparin strategy produced an incremental cost of CAN\$13,751 per QALY gained which is well within the reported Canadian threshold for adopting new medical interventions of CAN\$50,000 per QALY (Laupacis et al 1992). The authors concluded that dalteparin is a cost-effective treatment of cancer-related VTE.

\section{Conclusions}

Extended duration dalteparin has been clinically proven to reduce the risk of recurrent VTE without significantly increasing the risk of major bleeding in cancer patients with acute VTE. Additional benefits of dalteparin over conventional anticoagulant therapy in this patient population include ease of administration at the time of invasive procedures and chemotherapy-induced thrombocytopenia, lack of interaction with other medications and poor diet, and lack 
of need for laboratory monitoring. In general, dalteparin is well-tolerated by cancer patients, even those with end-stage malignancy.

\section{Disclosures}

The author has no conflicts of interest to disclose.

\section{References}

Alhenc-Gelas M, Jestin-Le Guernic C, Vitoux JF, et al. 1994. Adjusted versus fixed doses of the low-molecular-weight heparin fragmin in the treatment of deep vein thrombosis. Fragmin-Study Group. Thromb Haemost, 71:698-702.

Anonymous. 2006. Fragmin (dalteparin sodium) Product Monograph.

Aujesky D, Smith KJ, Cornuz J, et al. 2005. Cost-effectiveness of lowmolecular-weight heparin for secondary prophylaxis of cancer-related venous thromboembolism. Thromb Haemost, 93:592-99.

Bergqvist D. 2007. Risk of venous thromboembolism in patients undergoing cancer surgery and options for thromboprophylaxis. J Surg Oncol, 95:167-74.

Bergqvist D, Agnelli G, Cohen AT, et al. 2002. Duration of prophylaxis against venous thromboembolism with enoxaparin after surgery for cancer. N Engl J Med, 346:975-80.

Blom JW, Doggen CJ, Osanto S, et al. 2005. Malignancies, prothrombotic mutations, and the risk of venous thrombosis. JAMA, 293:715-22.

Blom JW, Vanderschoot JP, Oostindier MJ, et al. 2006. Incidence of venous thrombosis in a large cohort of 66,329 cancer patients: results of a record linkage study. J Thromb Haemost, 4:529-35.

Boneu B and de Moerloose P. 2001. How and when to monitor a patient treated with low molecular weight heparin. Semin Thromb Hemost, 27:519-22.

Bratt G, Tornebohm E, Widlund L, et al. 1986. Low molecular weight heparin (KABI 2165, Fragmin): pharmacokinetics after intravenous and subcutaneous administration in human volunteers. Thromb Res, 42:613-20.

Bromberg ME, Konigsberg WH, Madison JF, et al. 1995. Tissue factor promotes melanoma metastasis by a pathway independent of blood coagulation. Proc Natl AcadSci USA, 92:8205-9.

Buller HR, Agnelli G, Hull RD, et al. 2004. Antithrombotic therapy for venous thromboembolic disease: the Seventh ACCP Conference on Antithrombotic and Thrombolytic Therapy. Chest, 126:401S-28S.

Buller HR, van Doormaal FF, van Sluis GL, et al. 2007. Cancer and thrombosis: from molecular mechanisms to clinical presentations. $J$ Thromb Haemost, 5(Suppl 1):246-54.

Chew HK, Wun T, Harvey D, et al. 2006. Incidence of venous thromboembolism and its effect on survival among patients with common cancers. Arch Intern Med, 166:458-64.

Collignon F, Frydman A, Caplain H, et al. 1995. Comparison of the pharmacokinetic profiles of three low molecular mass heparins - dalteparin, enoxaparin and nadroparin - administered subcutaneously in healthy volunteers (doses for prevention of thromboembolism). Thromb Haemost, 73:630-40.

Couban S, Goodyear M, Burnell M, et al. 2005. Randomized placebo-controlled study of low-dose warfarin for the prevention of central venous catheterassociated thrombosis in patients with cancer. J Clin Oncol, 23:4063-9.

Cronin CG, Lohan DG, Keane M, et al. 2007. Prevalence and significance of asymptomatic venous thromboembolic disease found on oncologic staging CT. AJR Am J Roentgenol, 189:162-70.

Deitcher SR and Gomes MP. 2004. The risk of venous thromboembolic disease associated with adjuvant hormone therapy for breast carcinoma: a systematic review. Cancer, 101:439-49.

Deitcher SR, Kessler CM, Merli G, et al. 2006. Secondary prevention of venous thromboembolic events in patients with active cancer: enoxaparin alone versus initial enoxaparin followed by warfarin for a 180-day period. Clin Appl Thromb Hemost, 12:389-96.

Dranitsaris G, Vincent M, and Crowther M. 2006. Dalteparin versus warfarin for the prevention of recurrent venous thromboembolic events in cancer patients: a pharmacoeconomic analysis. Pharmacoeconomics, 24:593-607.
Dunn CJ and Jarvis B. 2000. Dalteparin: an update of its pharmacological properties and clinical efficacy in the prophylaxis and treatment of thromboembolic disease. Drugs, 60:203-37.

El Accaoui RN, Shamseddeen WA, and Taher AT. 2007. Thalidomide and thrombosis. A meta-analysis. Thromb Haemost, 97:1031-6.

ENOXACAN Study Group. 1997. Efficacy and safety of enoxaparin versus unfractionated heparin for prevention of deep vein thrombosis in elective cancer surgery: a double-blind randomized multicentre trial with venographic assessment. ENOXACAN Study Group. Br J Surg, 84:1099-103.

Fareed J, Leong WL, Hoppensteadt DA, et al. 2004. Generic low-molecularweight heparins: some practical considerations. Semin Thromb Hemost, 30:703-13.

Fiessinger JN, Lopez-Fernandez M, Gatterer E, et al. 1996. Once-daily subcutaneous dalteparin, a low molecular weight heparin, for the initial treatment of acute deep vein thrombosis. Thromb Haemost, 76:195-9.

Fricker JP, Vergnes Y, Schach R, et al. 1988. Low dose heparin versus low molecular weight heparin (Kabi 2165, Fragmin) in the prophylaxis of thromboembolic complications of abdominal oncological surgery. Eur J Clin Invest, 18:561-7.

Gale AJ and Gordon SG. 2001. Update on tumor cell procoagulant factors. Acta Haematol, 106:25-32.

Geerts WH, Pineo GF, Heit JA, et al. 2004. Prevention of venous thromboembolism: the Seventh ACCP Conference on Antithrombotic and Thrombolytic Therapy. Chest, 126:338S-400S

Gould MK, Dembitzer AD, Doyle RL, et al. 1999. Low-molecular-weight heparins compared with unfractionated heparin for treatment of acute deep venous thrombosis. A meta-analysis of randomized, controlled trials. Ann Intern Med, 130:800-9.

Grignani G and Maiolo A. 2000. Cytokines and hemostasis. Haematologica, 85:967-972.

Haddad TC and Greeno EW. 2006. Chemotherapy-induced thrombosis. Thromb Res, 118:555-68.

Handeland GF, Abildgaard U, Holm HA, et al. 1990. Dose adjusted heparin treatment of deep venous thrombosis: a comparison of unfractionated and low molecular weight heparin. Eur J Clin Pharmacol, 39:107-12.

Harenberg J, Huck K, Bratsch H, et al. 1990. Therapeutic application of subcutaneous low-molecular-weight heparin in acute venous thrombosis. Haemostasis, 20(Suppl 1):205-19.

Heit JA, Mohr DN, Silverstein MD, et al. 2000a. Predictors of recurrence after deep vein thrombosis and pulmonary embolism: a populationbased cohort study. Arch Intern Med, 160:761-8.

Heit JA, Silverstein MD, Mohr DN, et al. 2000b. Risk factors for deep vein thrombosis and pulmonary embolism: a population-based case-control study. Arch Intern Med, 160:809-15.

Herbst RS and Sandler AB. 2004. Non-small cell lung cancer and antiangiogenic therapy: what can be expected of bevacizumab? Oncologist, 9(Suppl 1):19-26.

Hirsh J. 2007. Risk of thrombosis with lenalidomide and its prevention with aspirin. Chest, 131:275-7.

Hirsh J and Raschke R. 2004. Heparin and low-molecular-weight heparin: the Seventh ACCP Conference on Antithrombotic and Thrombolytic Therapy. Chest, 126:188S-203S.

Holm HA, Ly B, Handeland GF, et al. 1986. Subcutaneous heparin treatment of deep venous thrombosis: a comparison of unfractionated and low molecular weight heparin. Haemostasis, 16(Suppl 2):30-7.

Hull JH and Hull PJ. 2003. Dalteparin compared with an oral anticoagulant for thromboprophylaxis in patients with cancer. $N$ Engl J Med, 349:1385-7.

Hull RD, Pineo GF, Brant RF, et al. 2006. Long-term low-molecular-weight heparin versus usual care in proximal-vein thrombosis patients with cancer. Am J Med, 119:1062-72.

Hurwitz H, Fehrenbacher L, Novotny W, et al. 2004. Bevacizumab plus irinotecan, fluorouracil, and leucovorin for metastatic colorectal cancer. N Engl J Med, 350:2335-42. 
Hutten BA, Prins MH, Gent M, et al. 2000. Incidence of recurrent thromboembolic and bleeding complications among patients with venous thromboembolism in relation to both malignancy and achieved international normalized ratio: a retrospective analysis. J Clin Oncol, $18: 3078-83$

Kabbinavar F, Hurwitz HI, Fehrenbacher L, et al. 2003. Phase II, randomized trial comparing bevacizumab plus fluorouracil (FU)/leucovorin (LV) with FU/LV alone in patients with metastatic colorectal cancer. $J$ Clin Oncol, 21:60-5.

Kakkar AK, Haas S, Wolf H, et al. 2005. Evaluation of perioperative fatal pulmonary embolism and death in cancer surgical patients: the MC-4 cancer substudy. Thromb Haemost, 94:867-71.

Karthaus M, Kretzschmar A, Kroning H, et al. 2006. Dalteparin for prevention of catheter-related complications in cancer patients with central venous catheters: final results of a double-blind, placebo-controlled phase III trial. Ann Oncol, 17:289-96.

Kearon C, Ginsberg JS, Julian JA, et al. 2006. Comparison of fixed-dose weight-adjusted unfractionated heparin and low-molecular-weight heparin for acute treatment of venous thromboembolism. JAMA, 296:935-42.

Kovacs MJ, Levine MN, Keeney M, et al. 2005. Anti-Xa effect of a low molecular weight heparin (dalteparin) does not accumulate in extended duration therapy for venous thromboembolism in cancer patients. Thromb Haemost, 93:1185-88.

Laupacis A, Feeny D, Detsky AS, et al. 1992. How attractive does a new technology have to be to warrant adoption and utilization? Tentative guidelines for using clinical and economic evaluations. CMAJ, 146:473-81.

Lee AY and Levine MN. 2003. Venous thromboembolism and cancer: risks and outcomes. Circulation, 107:I17-I21.

Lee AY, Levine MN, Baker RI, et al. 2003. Low-molecular-weight heparin versus a coumarin for the prevention of recurrent venous thromboembolism in patients with cancer. $N$ Engl J Med, 349:146-53.

Lee AY, Levine MN, Butler G, et al. 2006. Incidence, risk factors, and outcomes of catheter-related thrombosis in adult patients with cancer. J Clin Oncol, 24:1404-8.

Leizorovicz A, Simonneau G, Decousus H, et al. 1994. Comparison of efficacy and safety of low molecular weight heparins and unfractionated heparin in initial treatment of deep venous thrombosis: a meta-analysis. BMJ, 309:299-304.

Levine RL, McCollum D, and Hursting MJ. 2006. How frequently is venous thromboembolism in heparin-treated patients associated with heparininduced thrombocytopenia? Chest, 130:681-7.

Lindmarker P, Holmstrom M, Granqvist S, et al. 1994. Comparison of once-daily subcutaneous Fragmin with continuous intravenous unfractionated heparin in the treatment of deep vein thrombosis. Thromb Haemost, 72:186-90.

Luomanmaki K, Grankvist S, Hallert C, et al. 1996. A multicentre comparison of once-daily subcutaneous dalteparin (low molecular weight heparin) and continuous intravenous heparin in the treatment of deep vein thrombosis. J Intern Med, 240:85-92.

Meyer G, Brenot F, Pacouret G, et al. 1995. Subcutaneous low-molecularweight heparin fragmin versus intravenous unfractionated heparin in the treatment of acute non massive pulmonary embolism: an open randomized pilot study. Thromb Haemost, 74:1432-435.

Meyer G, Marjanovic Z, Valcke J, et al. 2002. Comparison of low-molecularweight heparin and warfarin for the secondary prevention of venous thromboembolism in patients with cancer: a randomized controlled study. Arch Intern Med, 162:1729-35.

Mismetti P, Laporte S, Darmon JY, et al. 2001. Meta-analysis of low molecular weight heparin in the prevention of venous thromboembolism in general surgery. Br J Surg, 88:913-30.

Monreal M, Zacharski L, Jimenez JA, et al. 2004. Fixed-dose low-molecular-weight heparin for secondary prevention of venous thromboembolism in patients with disseminated cancer: a prospective cohort study. J Thromb Haemost, 2:1311-15.

Noble SI and Finlay IG. 2005. Is long-term low-molecular-weight heparin acceptable to palliative care patients in the treatment of cancer related venous thromboembolism? A qualitative study. Palliat Med, 19:197-201
Noble SI, Hood K, and Finlay IG. 2007. The use of long-term low-molecular weight heparin for the treatment of venous thromboembolism in palliative care patients with advanced cancer: a case series of sixty two patients. Palliat Med, 21:473-6.

Otten HM, Mathijssen J, ten CH, et al. 2004. Symptomatic venous thromboembolism in cancer patients treated with chemotherapy: an underestimated phenomenon. Arch Intern Med, 164:190-4.

Prandoni P, Falanga A, and Piccioli A. 2005. Cancer and venous thromboembolism. Lancet Oncol, 6:401-10.

Prandoni P, Lensing AW, Piccioli A, et al. 2002. Recurrent venous thromboembolism and bleeding complications during anticoagulant treatment in patients with cancer and venous thrombosis. Blood, 100:3484-8.

Quinlan DJ, McQuillan A, and Eikelboom JW. 2004. Low-molecular-weight heparin compared with intravenous unfractionated heparin for treatment of pulmonary embolism: a meta-analysis of randomized, controlled trials. Ann Intern Med, 140:175-83.

Rabbat CG, Cook DJ, Crowther MA, et al. 2005. Dalteparin thromboprophylaxis for critically ill medical-surgical patients with renal insufficiency. $J$ Crit Care, 20:357-63.

Rajkumar SV and Blood E. 2006. Lenalidomide and venous thrombosis in multiple myeloma. N Engl J Med, 354:2079-80.

Rak J, Yu JL, Luyendyk J, et al. 2006. Oncogenes, trousseau syndrome, and cancer-related changes in the coagulome of mice and humans. Cancer Res, 66:10643-6.

Rasmussen MS, Jorgensen LN, Wille-Jorgensen P, et al. 2006. Prolonged prophylaxis with dalteparin to prevent late thromboembolic complications in patients undergoing major abdominal surgery: a multicenter randomized open-label study. J Thromb Haemost, 4:2384-90.

Rasmussen MS, Willie-Jorgensen P, and Jorgensen LN. 2005. Extended outof-hospital low-molecular-weight heparin prophylaxis against venous thromboembolism in patients after cancer operations: a meta-analysis. J Thromb Haemost, 3(Suppl 1):Abstract P2213.

Rickles FR, Patierno S, and Fernandez PM. 2003. Tissue factor, thrombin, and cancer. Chest, 124:58S-68S.

Rugo HS. 2004. Bevacizumab in the treatment of breast cancer: rationale and current data. Oncologist, 9(Suppl 1):43-49.

Sallah S, Wan JY, and Nguyen NP. 2002. Venous thrombosis in patients with solid tumors: determination of frequency and characteristics. Thromb Haemost, 87:575-9.

Shah MA, Ilson D, and Kelsen DP. 2005. Thromboembolic events in gastric cancer: high incidence in patients receiving irinotecan- and bevacizumab-based therapy. J ClinOncol, 23:2574-6.

Simoneau G, Bergmann JF, Kher A, et al. 1992. Pharmacokinetics of a low molecular weight heparin [Fragmin] in young and elderly subjects. Thromb Res, 66:603-7.

Sorensen HT, Mellemkjaer L, Olsen JH, et al. 2000. Prognosis of cancers associated with venous thromboembolism. $N$ Engl J Med, 343:1846-50.

Tagalakis V, Levi D, Agulnik JS, et al. 2007. High risk of deep vein thrombosis in patients with non-small cell lung cancer: a cohort study of 493 patients. J Thorac Oncol, 2:729-34.

Verso M, Agnelli G, Bertoglio S, et al. 2005. Enoxaparin for the prevention of venous thromboembolism associated with central vein catheter: a double-blind, placebo-controlled, randomized study in cancer patients. J Clin Oncol, 23:4057-62.

von Tempelhoff GF, Dietrich M, Hommel G, et al. 1996. Blood coagulation during adjuvant epirubicin/cyclophosphamide chemotherapy in patients with primary operable breast cancer. J Clin Oncol, 14:2560-8.

von Tempelhoff GF, Niemann F, Schneider DM, et al. 1998. Blood rheology during chemotherapy in patients with ovarian cancer. Thromb Res, 90:73-82.

Wells PS, Anderson DR, Rodger MA, et al. 2005. A randomized trial comparing 2 low-molecular-weight heparins for the outpatient treatment of deep vein thrombosis and pulmonary embolism. Arch Intern Med, 165:733-8.

Zonder JA, Barlogie B, Durie BG, et al. 2006. Thrombotic complications in patients with newly diagnosed multiple myeloma treated with lenalidomide and dexamethasone: benefit of aspirin prophylaxis. Blood, 108:403-4. 
